\title{
Management of invasive ductal carcinoma in hypermobile Ehlers-Danlos syndrome: a case report
}

\author{
Nikita Deshpande ${ }^{1 \wedge}$, Stephanie Murti ${ }^{2}$, Rashi Singh ${ }^{3}$, Cameron Coker $^{4}$, Sonali Rudra ${ }^{5}$, Nitika Paudel ${ }^{5}$, \\ Kenneth L. Fan ${ }^{6}$, Ian T. Greenwalt ${ }^{4}$ \\ ${ }^{1}$ Georgetown University School of Medicine, Washington, DC, USA; ${ }^{2}$ Kansas City University School of Medicine, Kansas City, MO, USA; \\ ${ }^{3}$ Department of Surgery, University Hospitals/Case Western Reserve University, Cleveland, OH, USA; ${ }^{4}$ Department of Surgery, MedStar \\ Georgetown University Hospital, Washington, DC, USA; ${ }^{5}$ Department of Radiation Oncology, MedStar Georgetown University Hospital, \\ Washington, DC, USA; ${ }^{\circ}$ Department of Plastic Surgery, MedStar Georgetown University Hospital, Washington, DC, USA \\ Correspondence to: Nikita Deshpande. 3900 Reservoir Road NW, Washington, DC 20007, USA. Email: nd421@georgetown.edu.
}

\begin{abstract}
Literature is sparse regarding the management and long-term outcomes of breast cancer in patients with Ehlers-Danlos syndrome (EDS). Of the EDS subtypes, hypermobile Ehlers-Danlos Syndrome (hEDS) is associated with cardiovascular dysautonomia which manifests as spontaneous episodes of tachycardia and hypotension. Given this clinical autonomic system impact, hEDS is known to have significant intraoperative risk and postoperative complications. However, outcomes of hEDS patients have not been specifically studied in the field of breast cancer surgery. Here we present a case of a 62-year-old female with hEDS and node-positive invasive ductal breast carcinoma. Given the patient's medical history of hEDS, close attention was given to the patient's intraoperative vital signs and predisposition for poor wound healing. The patient underwent left Goldilocks mastectomy with left axillary lymph node dissection. Due to cardiac comorbidities, she was not a candidate for neoadjuvant or adjuvant chemotherapy. The patient tolerated adjuvant radiation and endocrine therapy without side effects, and has remained free of local, regional, and distant cancer recurrence following treatment. This case report highlights a literature gap in the surgical and radiation therapy management of breast cancer in patients with hEDS. Although breast surgery and radiation therapy in patients with invasive breast cancer and hEDS can be a safe management option, we discuss how perioperative complications must be cautiously navigated and how treatment must be tailored to individuals' specific hEDS variant to ensure optimal patient safety and positive long-term outcomes.
\end{abstract}

Keywords: Breast cancer; hypermobile Ehlers-Danlos syndrome (hEDS); autonomic dysfunction; case report

Submitted Jun 08, 2021. Accepted for publication Aug 13, 2021.

doi: $10.21037 /$ gs-21-380

View this article at: https://dx.doi.org/10.21037/gs-21-380

\section{Introduction}

Ehlers-Danlos syndrome (EDS) is a rare disease that affects anywhere between $0.02-0.04 \%$ of births (1) and can either be inherited in an autosomal dominant or recessive fashion. There are four major inherited subtypes of autosomal dominant EDS including: hypermobile, vascular, arthrochalasis, and classic. The two main autosomal recessive types include: dermatosparaxis and kyphoscoliotic (2). These six subtypes comprise a group of collagen vascular disorders which derive from gene mutations in fibrillar collagen-encoding genes, or in posttranslational modifying enzymes. Of the EDS subtypes, hypermobile EDS (hEDS) is the most common connective tissue disease variant with community prevalence affecting

^ ORCID: 0000-0001-6447-043X. 


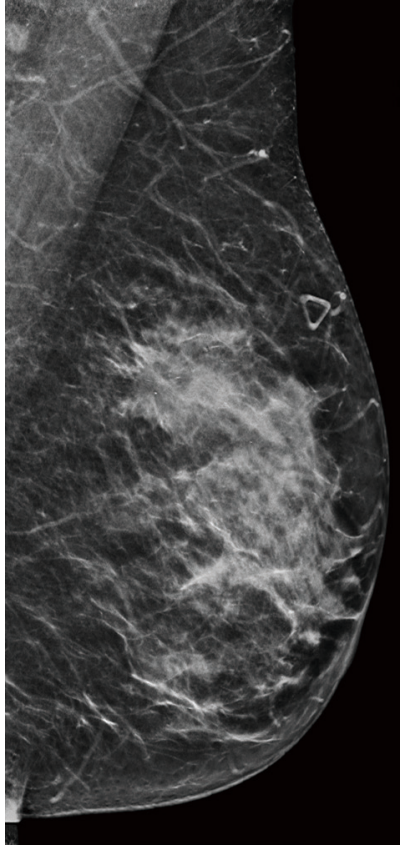

Figure 1 Mediolateral oblique mammogram showing a $2.9 \mathrm{~cm}$ $\times 2.5 \mathrm{~cm} \times 2.7 \mathrm{~cm}$ mass in the upper outer quadrant of the left breast-located about $8 \mathrm{~cm}$ from the nipple-and enlarged left axillary adenopathy.

$0.007-0.01 \%$ of births (1). hEDS is further associated with cardiovascular dysautonomia which manifests as spontaneous episodes of tachycardia and hypotension (3). Despite the prevalence and autonomic challenges of hEDS, surgical management guidelines for patients with hEDS have not been widely researched (4). Patients with breast cancer and hEDS further pose unique management challenges, and to date only a small number of cases have been described (5). We present the following case in accordance with the CARE reporting checklist (available at https://dx.doi.org/10.21037/gs-21-380).

\section{Case presentation}

We report a case of a 62-year-old Caucasian woman who was referred to our institution after palpating a mass in her left breast. The patient was postmenopausal and nulliparous. Her medical history was notable for hEDS with microvascular disease and autonomic dysfunction, cardiac history of spontaneous coronary artery dissection of the left anterior descending artery (LAD) treated with percutaneous intervention and myocardial infarction, and musculoskeletal

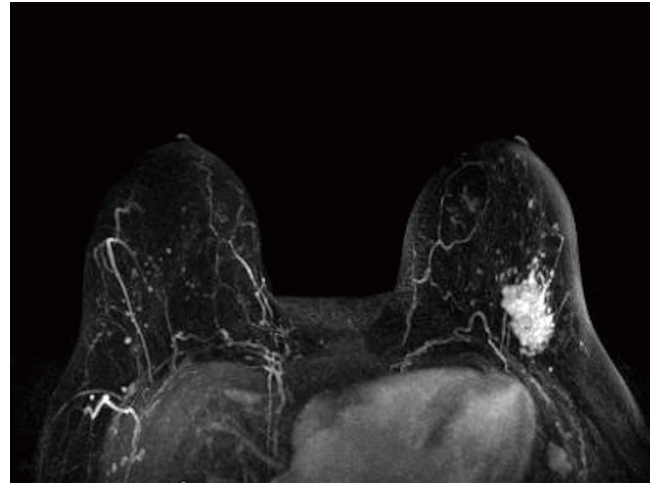

Figure 2 Bilateral magnetic resonance imaging (MRI) showing normal right breast, and a $2.9 \mathrm{~cm} \times 3.7 \mathrm{~cm} \times 4.1 \mathrm{~cm}$ oval mass in the left breast at 2 o'clock posterior depth. Dominant satellite nodule about $6 \mathrm{~mm}$ in size, is located $1.5 \mathrm{~cm}$ from carcinoma within the lower outer quadrant of the left breast and is suspicious for malignancy.

history significant for adhesive capsulitis and frozen shoulder. Physical exam revealed a $3 \mathrm{~cm}$ mass in the upper outer quadrant of the left breast at 2 o'clock with no nipple retraction or discharge, and a palpable left axillary lymph node. Mammogram (Figure 1) showed a $2.9 \mathrm{~cm} \times 2.5 \mathrm{~cm}$ $\times 2.7 \mathrm{~cm}$ mass in the upper outer quadrant of the left breast about $8 \mathrm{~cm}$ from the nipple with enlarged left axillary adenopathy. Ultrasound-guided core needle biopsy returned grade II invasive ductal carcinoma (IDC) with ductal carcinoma in situ (DCIS) in the left breast, and metastatic carcinoma of the left axillary lymph node. Estrogen receptor (ER) and progesterone receptor (PR) status of the tumor revealed $\mathrm{ER}>90 \%, \mathrm{PR}>90 \%$, Her2/neu negative, Ki-67 73-81\%, and p53 90\%. Breast magnetic resonance imaging (MRI) was obtained (Figure 2), demonstrating $6 \mathrm{~mm}$ satellite nodules located $1.5 \mathrm{~cm}$ away from the cancer in the lower outer quadrant of the left breast. Positron emission tomography/computed tomography (PET/CT) showed increased uptake in the left breast and axilla with no distant metastatic disease.

The preoperative treatment plan involved cardiac and anesthesia clearance from an EDS specialist given the patient's history of hEDS, coronary dissection, and dysautonomia, as well as a physical medicine and rehabilitation evaluation for lymphedema screening given the patient's history of adhesive capsulitis and frozen shoulder. Surgical treatment plan involved upfront surgery via simple mastectomy with preservation of the inferior dermoglandular pedicle (Goldilocks mastectomy), axillary lymph node 


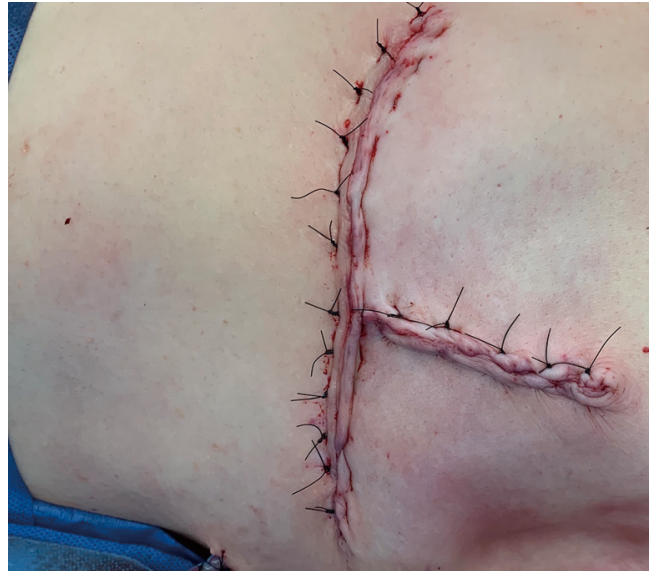

Figure 3 Postoperative photograph demonstrating overall design of left breast Goldilocks mastectomy.

dissection, and plastic surgery wound closure. Goldilocks mastectomy was performed by de-epithelializing the dermal mastectomy flaps and raising the pedicle within the mastectomy plane. Following simple mastectomy, the pedicle was folded over to allow for proper inset and overlying skin was closed (Figure 3). As per patient preference, the opposite breast was not reduced. Neoadjuvant therapy was not pursued due to cardiotoxicity concerns and potential for decreased chemosensitivity with the patient's ER/PR positive, HER2neu negative cancer profile. Upfront surgery was also pursued to obtain complete pathologic axillary staging as axillary radiation could potentially be avoided for N1 disease with adequate axillary dissection.

Final postoperative pathology revealed $4 \mathrm{~cm}$ grade II IDC with lymphovascular invasion, 5/17 lymph nodes positive, extranodal extension present, and ER+, PR+, HER2neu negative receptor status. Final pathologic stage was IIIA (pT2 $\mathrm{pN} 2 \mathrm{a} \mathrm{pM} 0$ ). No immediate post-operative complications occurred, and the patient tolerated surgery well.

With surgery and adjuvant radiation therapy, the patient is expected to have good local control. Given concerns for radiation toxicity to a previously stented $\mathrm{LAD}$, the heart and chest wall were completely excluded from the radiation field. The left axilla and supraclavicular lymph nodes were instead targeted with proton therapy. Excluding the chest wall decreased potential toxicity to the coronary arteries, however, this placed the patient at higher risk for chest wall recurrence. Adjuvant endocrine therapy is expected to improve survival and decrease chance of systemic recurrence; however, the patient's inability to receive adjuvant chemotherapy and specific cancer characteristics of ER/PR positivity, HER2neu negativity, and high Ki67 place her at increased risk for recurrence and decreased overall survival.

The patient experienced a four-to-six-week delay in initiating adjuvant radiation due to concerns over additional therapy and limited left upper extremity range of motion due to postoperative pain and history of frozen shoulder. Adjuvant radiation was recommended given the patient's N2 disease status with aggressive characteristics (lymphovascular invasion and extranodal extension). Undissected axillary lymph nodes-levels II, III and the supraclavicular fossa-were treated with proton therapy. The chest wall and internal mammary lymph nodes were omitted given concerns for cardiac toxicity. The heart was inferior to the irradiated region and subsequently received a negligible dose of radiation therapy. Overall, the patient tolerated adjuvant radiation therapy well with minor sequela of mild fatigue. Although the patient experienced grade II dermatitis one month after beginning radiation therapy, her skin reaction completely resolved over the next two months with use of topical steroid and moisturizing creams. As of the patient's latest clinic visit in 2021, there have been no long-term skin changes.

The patient was further recommended adjuvant endocrine therapy with anastrozole and has tolerated endocrine therapy since initiation with no side effects. Adjuvant chemotherapy was not recommended given the patient's lower oncotype score of 19, history of hEDS, and significant cardiac comorbidities. Although our patient was not given neoadjuvant or adjuvant chemotherapy, it is important to note that diagnosis of hEDS by itself does not influence the decision for upfront chemotherapy administration in phenotypically appropriate patients with triple negative cancer or HER2 positive cancer. hEDS patients who lack significant cardiac comorbidities and have phenotypically appropriate cancer would likely receive neoadjuvant chemotherapy. Furthermore, hEDS patients with high oncotype scores would likely receive adjuvant chemotherapy to reduce the risk of future recurrence.

Following Goldilocks mastectomy and radiation therapy, the patient remains free of local, regional, and distant cancer recurrence as of her latest clinic visit in 2021. All procedures performed in studies involving human participants were in accordance with the ethical standards of the institutional research committee and with the Helsinki Declaration (as revised in 2013). Written informed consent was obtained from the patient for publication of this case report and accompanying images. A copy of the written consent is 
available for review by the editorial office of this journal.

\section{Discussion}

Collagen is an essential component of the wound healing process. Patients with EDS experience wound closure delay because they express a mutated variant of collagen that leads to biosynthesis abnormalities and general tissue fragility (5). This renders patients with EDS at risk of perioperative wound dehiscence, prolonged wound healing time, surgical-site infection, and adjuvant therapy delay (4).

Despite the clear relationship between EDS and poor wound healing, few guidelines dictate proper surgical wound care in EDS patients. One leading thought based on limited available literature is that advanced therapeutic wound dressings may accelerate wound healing in this patient population (5).

Advanced therapeutic wound dressings are produced using a variety of materials, impregnation formulations, and drug-delivery systems (6). Baik et al. (7) illustrate that use of a mixed material, alginate-based collagen dressing precipitated wound healing in a patient with EDS following partial skin necrosis and delayed closure of a lateral foot wound repaired in primary approximation. This case report is promising, however, further clinical studies are needed to inform evidence-based utilization of advanced therapeutic wound dressings in EDS.

Considering our patient's EDS-driven predisposition to poor wound healing, the two main postoperative concerns were to design a tolerable breast reconstruction plan, and to design a tolerable adjuvant radiation therapy plan that could properly manage the patient's risks.

Breast reconstruction in EDS is a challenging endeavor because few guidelines outline management priorities for these patients $(4,5)$. As a result, clinicians are left to develop breast reconstruction plans using best-practice knowledge. Our surgical team opted for breast reconstruction via Goldilocks mastectomy to minimize the patient's risk for wound healing complications.

The Goldilocks mastectomy has been widely used as an alternative breast reconstruction option for individuals who are poor candidates for traditional breast reconstruction due to medical comorbidities such as connective tissue disorders (8). First described by Richardson and $\mathrm{Ma}$ (8), the Goldilocks mastectomy recreates the breast mound from mastectomy flaps as a single-stage reconstruction. The procedure typically involves local contouring of autologous breast tissue, created by preserving and de-epithelializing the residual vascularized mastectomy flaps. Due to skin breakdown and de-epithelialization of the dermal layer, tissue expander can be placed at the time of mastectomy and later exchanged for implant at the time of reconstruction. Of note, this approach can be modified for patients with EDS through the addition of a dermal substitute or acellular dermal matrix $(\mathrm{ADM})$ to the de-epithelialized layer. These substances act as a barrier to skin breakdown, and thus reinforce the breast tissue pocket to eventually support an implant (9).

Although the traditional Goldilocks approach is typically indicated for patients receiving staged implant reconstructions, the single-stage Goldilocks procedure with $\mathrm{ADM}$ reinforcement is an excellent reconstruction option for EDS patients that has not been extensively discussed in breast cancer surgery literature. In particular, the de-epithelialized dermal layer is beneficial for patients with EDS because it acts as a barrier to skin breakdown. The Goldilocks mastectomy should be increasingly studied as a potential breast reconstructive option for patients with comorbidities that predispose to poor wound healing (8), and we hope this case report helps increase the utilization of Goldilocks mastectomy in patients with connective tissue disorders such as EDS.

Beyond impacting reconstruction options, EDS further impacts the delivery of radiation therapy. In patients with underlying conditions that predispose to poor wound healing, radiation can lead to increased wound healing complications $(10,11)$. As a result, adjuvant radiation therapy must be designed with care in patients with EDS. Literature review demonstrates a paucity of studies that explore the role of radiation therapy in EDS. Of existing papers that have been published, there is a mixed consensus on the efficacy and risks of radiation therapy for patients with EDS. Two case reports suggest that radiation therapy may be a tolerable option in patients who are able withstand the side-effect profile $(12,13)$. However, one case report (14) cautions against the use of radiation therapy in EDS.

In particular, Chau and Chen (12) present a case report involving a 42 -year-old female with type III EDS who received adjuvant radiation therapy following breastconservation surgery for a hormone-receptor stage II mucinous/colloid carcinoma. Although the patient had encouraging long-term post-radiation outcomes, immediately after radiation treatment she developed three weeks of severe, grade III radiation dermatitis. The dermatitis resolved with local wound care; however, the authors deemed the patient's degree of dermatitis as comparatively worse than that experienced by patients 
without EDS. Despite the patient's transient dermatitis, radiation therapy was holistically viewed as a successful option in managing the patient's postoperative risk of cancer recurrence. Another case report by Falchook \& Zagar (13) describes how a 71-year-old female with oligometastatic lung cancer tolerated external beam radiation with concurrent chemotherapy to the lung/mediastinum followed by stereotactic radiosurgery (SRS) to the brain well without vascular events and achieved appropriate local disease control. These case reports suggest that radiation therapy could be a feasible option for EDS patients.

In contrast to the above papers which present a favorable view of radiation therapy in EDS, a case report by Holodny et al. (14) shares how a 62-year-old female with EDS and metastatic breast cancer with numerous brain metastases developed multiple aneurysms throughout her circle of Willis following the tissue-destructive effects of whole-brain radiation therapy, and eventually died of an aneurysmal rupture. This case report highlights that radiation therapy may not be optimal for all EDS patients.

Despite the conflicting publications on the use of adjuvant radiation in EDS, it is important to note that a broader range of studies which specifically focus on the use of radiation therapy in patients with EDS is needed to guide future therapy (15). Our care team opted for adjuvant radiation therapy, and as of the patient's latest clinic visit in 2021, she has not experienced cancer recurrence since treatment initiation. However, the decision to initiate adjuvant radiation therapy was difficult given the lack of guidelines governing indications or contraindications for radiation therapy in EDS. We hope this case report is of value to future clinicians involved in breast cancer management of patients with inherited, collagen-based connective tissue diseases.

\section{Conclusions}

In summary, EDS comprises a collection of hereditary disorders characterized by connective tissue fragility. Surgical and radiation therapy management of patients with hEDS and breast cancer pose a unique challenge as these patients are at increased risk of intraoperative autonomic fluctuation and postoperative wound healing complications. Additionally, breast reconstruction and design of adjuvant radiation must manage these patients' predispositions to wound and vascular complications. Our case presentation of hEDS and breast cancer is of value given our patient's medical history of hEDS, associated dysautonomia, and cardiac comorbidities which required meticulous preoperative planning and thoughtful adjuvant recommendations. Current literature does not extensively cover surgical or adjuvant management of patients with breast cancer and EDS. This case report helps bring awareness to the current literature gap while also contributing to our group's experience with management of EDS breast cancer patients. We hope this case report will help with establishment of clinical guidelines for cancer management in patients with inherited, collagen-related connective tissue diseases like EDS.

\section{Acknowledgments}

We are grateful to the multidisciplinary breast cancer team at MedStar Georgetown University Hospital/Lombardi Cancer Center for care and support of this patient and case report.

Funding: None.

\section{Footnote}

Reporting Checklist: The authors have completed the CARE reporting checklist. Available at https://dx.doi. org/10.21037/gs-21-380

Peer Review File: Available at https://dx.doi.org/10.21037/ gs-21-380

Conflicts of Interest: All authors have completed the ICMJE uniform disclosure form (available at https://dx.doi. org/10.21037/gs-21-380). The authors have no conflicts of interest to declare.

Etbical Statement: The authors are accountable for all aspects of the work in ensuring that questions related to the accuracy or integrity of any part of the work are appropriately investigated and resolved. All procedures performed in studies involving human participants were in accordance with the ethical standards of the institutional research committee and with the Helsinki Declaration (as revised in 2013). Written informed consent was obtained from the patient for publication of this case report and accompanying images. A copy of the written consent is available for review by the editorial office of this journal.

Open Access Statement: This is an Open Access article distributed in accordance with the Creative Commons 
Attribution-NonCommercial-NoDerivs 4.0 International License (CC BY-NC-ND 4.0), which permits the noncommercial replication and distribution of the article with the strict proviso that no changes or edits are made and the original work is properly cited (including links to both the formal publication through the relevant DOI and the license). See: https://creativecommons.org/licenses/by-nc-nd/4.0/.

\section{References}

1. Ehlers Danlos Syndromes [Internet]. Danbury (CT): NORD (National Organization for Rare Disorders). c2021 - [cited 2021 Jul 20]. Available online: https://rarediseases. org/rare-diseases/ehlers-danlos-syndrome/

2. Byers PH, Murray ML. Heritable collagen disorders: the paradigm of the Ehlers-Danlos syndrome. J Invest Dermatol. 2012;132:E6-11.

3. Hakim A, O'Callaghan C, De Wandele I, et al. Cardiovascular autonomic dysfunction in Ehlers-Danlos syndrome-Hypermobile type. Am J Med Genet C Semin Med Genet 2017;175:168-74.

4. Wiesmann T, Castori M, Malfait F, et al. Recommendations for anesthesia and perioperative management in patients with Ehlers-Danlos syndrome(s). Orphanet J Rare Dis 2014;9:109.

5. Joseph AW, Joseph SS, Francomano CA, et al. Characteristics, Diagnosis, and Management of EhlersDanlos Syndromes: A Review. JAMA Facial Plast Surg 2018;20:70-5.

6. Boateng J, Catanzano O. Advanced Therapeutic Dressings for Effective Wound Healing--A Review. J Pharm Sci

Cite this article as: Deshpande N, Murti S, Singh R, Coker C, Rudra S, Paudel N, Fan KL, Greenwalt IT. Management of invasive ductal carcinoma in hypermobile Ehlers-Danlos syndrome: a case report. Gland Surg 2021;10(9):2861-2866. doi: $10.21037 /$ gs-21-380
2015; 104:3653-80.

7. Baik BS, Lee WS, Park KS, et al. Treatment of the wide open wound in the Ehlers-Danlos syndrome. Arch Craniofac Surg 2019;20:130-3.

8. Richardson H, Ma G. The Goldilocks mastectomy. Int J Surg 2012;10:522-6.

9. Schuster RH, Rotter S, Boonn W, et al. The use of tissue expanders in immediate breast reconstruction following mastectomy for cancer. Br J Plast Surg $1990 ; 43: 413-8$.

10. Haubner F, Ohmann E, Pohl F, et al. Wound healing after radiation therapy: review of the literature. Radiat Oncol 2012;7:162.

11. Payne WG, Naidu DK, Wheeler CK, et al. Wound healing in patients with cancer. Eplasty 2008;8:e9.

12. Chau T, Chen AY. Successful case of breast conservation radiotherapy in a patient with type III Ehlers-Danlos syndrome. Breast J 2018;24:221-2.

13. Falchook AD, Zagar TM. A case report of stereotactic radiosurgery in a patient with Ehlers-Danlos syndrome. Rep Pract Oncol Radiother 2013;18:241-4.

14. Holodny AI, Deck M, Petito CK. Induction and subsequent rupture of aneurysms of the circle of Willis after radiation therapy in Ehlers-Danlos syndrome: a plausible hypothesis. AJNR Am J Neuroradiol 1996;17:226-32.

15. Tinkle B, Castori M, Berglund B, et al. Hypermobile Ehlers-Danlos syndrome (a.k.a. Ehlers-Danlos syndrome Type III and Ehlers-Danlos syndrome hypermobility type): Clinical description and natural history. Am J Med Genet C Semin Med Genet 2017;175:48-69. 